\title{
Diagnosis of Lynch syndrome before colorectal resection: does it matter?
}

\author{
A. Noll ${ }^{1}$ P. J. Parekh ${ }^{2}$ J. J. Karlitz ${ }^{2}$
}

Received: 22 January 2016/ Accepted: 22 January 2016/Published online: 19 February 2016

(C) Springer-Verlag Italia Srl 2016

Lynch syndrome (LS), also known as hereditary nonpolyposis colorectal cancer (HNPCC), is an autosomal dominant genetic condition which predisposes patients to several different types of cancer, most notably colorectal cancer [1]. LS can be implicated in up to $5 \%$ of colorectal cancers (CRC), making it the most common form of hereditary CRC [2]. However, in patients with early-onset CRC this number can be up to $17 \%$ [3]. There has been an increased focus on early-onset $\mathrm{CRC}$ in general due to recently demonstrated rising $\mathrm{CRC}$ incidence rates in this group, although the underlying pathogenic factors are not completely understood [4]. Timing of LS diagnosis in suspected patients is critical, as early identification can facilitate management decisions regarding surgical intervention, colonoscopic surveillance and screening for extracolonic cancers. In addition, expedited germline genetic testing can be offered to at-risk family members.

Recent guidelines suggest that all newly diagnosed CRCs, regardless of patient age, can be considered for evaluation for mismatch repair deficiency by microsatellite instability (MSI) and/or immunohistochemistry (IHC) testing for mismatch repair proteins [5]. Germline genetic testing can follow in order to confirm LS and assess for specific underlying mutations. When surgery is indicated, the aforementioned testing should ideally occur preoperatively so that results can assist planning and decision

\section{J. J. Karlitz \\ jkarlitz@tulane.edu}

1 School of Medicine, Tulane University, Tulane Avenue, New Orleans, LA 70112, USA

2 Division of Gastroenterology, Department of Internal Medicine, School of Medicine, Tulane University, 1430 Tulane Avenue, New Orleans, LA 70112, USA making in the operating room [5]. Due to high rates of metachronous CRC in LS patients undergoing partial colon resections (16\% at 10 years, $41 \%$ at 20 years and $62 \%$ at 30 years), even in those undergoing colonoscopic surveillance, colectomy with ileorectal anastomosis is recommended $[5,6]$. Studies have demonstrated the rate of metachronous lesions to drop significantly (postoperative risk is $0-3.4 \%$ ) when the recommended surgical intervention was performed [6, 7]. Given this expanding appreciation for the role that genetic analysis may play in CRC surgical and medical management, the expectations would be for a rise in genetic evaluation, unfortunately the reality seems far from it.

A recent study utilizing CDC comparative effectiveness research data conducted by our group evaluated the frequency of MSI and IHC testing in early-onset CRC patients and availability of testing results preoperatively [8]. The study, which was the first population-based study in the USA to evaluate LS screening practices, took place in Louisiana, which was recently demonstrated to have one of the highest incidence rates of CRC in the USA and very high rates of early-onset CRC, particularly in certain regions [9]. Data collected from the Louisiana Tumor Registry totaling 274 patients statewide $\leq 50$ years of age and diagnosed with CRC in 2011 were analyzed. They found that in this young, high-risk population in which LS screening rates would be expected to be the highest, MSI and/or IHC testing was performed in only $23 \%$ of patients with abnormalities found in $21.7 \%$ of cases. Additionally, of those with abnormal IHC staining patterns, results were suggestive of LS in $87.5 \%$ of cases highlighting the highrisk nature of the early-onset CRC population. Lastly, they found that these results were available preoperatively only $16.9 \%$ of the time due to testing being ordered infrequently on preoperative colonoscopy specimens or results 
returning after surgery took place. Hence due to the potential of LS being diagnosed postoperatively or not being diagnosed at all, the potential exists for patients to undergo segmental resection, which could leave them vulnerable to developing metachronous lesions. A strength of this study was the fact that it was population-based, representing care of all early-onset CRC patients in the state at 61 distinct healthcare facilities.

The implications of not identifying LS in the proband extend beyond the index cancer, namely screening for extra-colonic cancers and inadequate LS screening in atrisk family members. For example, with regard to the former, females with LS are at significantly higher risk for developing uterine cancer (upwards of $60 \%$ lifetime risk), and thus, early diagnosis is imperative for the purposes of screening and/or early prophylactic surgical intervention, e.g., total abdominal hysterectomy with bilateral salpingooophorectomy [10].

Although gaps in LS management have been demonstrated, the root cause needs further investigation. The aforementioned study examined several testing factors associated with an increased likelihood of undergoing LS screening including family history of CRC, residence in an urban setting and receiving care at a comprehensive cancer center [8]. The positive effect of family history may be obvious, but what can be made of urban location and treatment center influence? One can deduce that most likely there are multiple factors at play, namely access to more experienced specialists and increased availability of genetic testing and counseling. Moreover, it was found that the majority of tumor analyses ordered originated from only a few centers, suggesting a clustering of hereditary CRC specialists or perhaps the positive impact that a few influential providers can have on the approaches of their colleagues. No matter the reasoning, these results suggest that a targeted intervention on provider education at underperforming centers may be an effective means at closing the gap.

Overall, to make sense of this gap between recommended best practices and their current implementation, the problem can most productively be divided into two separate issues: first, the overall low rate of MSI and/or IHC testing and second, the rarity of having such results available prior to surgery. To begin, providers simply may not be well versed in interpreting results from MSI and/or IHC testing and subsequent germline testing, or may not have access to genetic counseling, all of which can prevent test ordering. For physicians who do not regularly manage hereditary cancer syndromes, the value of these tests for specific high-risk populations may be minimized. As a result, not only might overall rates of testing be decreased, but surgery may take place prior to the return of results which could help explain the second, previously raised issue. Likewise, another barrier to accessing testing results preoperatively may be physicians' (or patients') hesitance in the face of malignancy to wait several weeks for a protracted, two-step process of MSI and/or IHC testing on a colonoscopic specimen followed by germline genetic testing. In addition, patient and provider concerns regarding bowel function and quality of life after more extensive surgery may limit the performance of more extensive surgery even in the presence of abnormal testing. Those undergoing more extensive colonic resection may potentially experience increased stool frequency with a potential impact on quality of life [11]. However, this must be balanced with reduction in CRC risk that is achieved with more extensive surgery. The aforementioned factors, when considered in conjunction with the potential emotional and psychological concerns of the risk of passing a hereditary condition to family members, can potentially explain hesitation to proceed with genetic valuation.

Each potential barrier needs to be further explored as to better understand the factors involved in LS screening implementation so that testing rates can be maximized going forward. Genetic counseling is critical to incorporate into the process as it not only benefits the proband, but facilitates screening of at-risk family members. In the end, a streamlined process that not only maximizes LS screening rates but optimizes the timing of availability of test results will benefit patients and physicians alike. Importantly, a protocol-driven process needs to be implemented so that MSI and/or IHC analysis and germline genetic testing are processed as quickly as possible so that results are made available preoperatively. This will likely involve a multifactorial approach integrating providers, insurance carriers and genetic testing companies. Even if in certain cases patients and physicians are uncomfortable with more extensive colonic resection, at least test results can be in place preoperatively so a detailed risk/benefit discussion can take place. As a streamlined process comes to fruition, patients will become more in tune to their disease process and physicians will have all the necessary information prior to implementing both medical and surgical management decisions.

\section{Compliance with ethical standards}

Conflict of interest The authors declare that they have no conflict of interest.

Ethical approval The research performed by Dr. Karlitz referenced in this article met all ethical standards set forth by the accrediting body.

Informed consent For this editorial informed consent is not required. 


\section{References}

1. Lynch HT, Shaw MW, Magnuson CW, Larsen AL, Krush AJ (1966) Hereditary factors in cancer. Study of two large midwestern kindreds. Arch Intern Med 117:206-212

2. Jasperson KW, Tuohy TM, Neklason DW, Burt RW (2010) Hereditary and familial colon cancer. Gastroenterology 138:2044-2058

3. Southey MC, Jenkins MA, Mead L et al (2005) Use of molecular tumor characteristics to prioritize mismatch repair gene testing in early-onset colorectal cancer. J Clin Oncol 23:6524-6532

4. Bailey CE, Hu CY, You YN et al (2014) Increasing disparities in the age-related incidences of colon and rectal cancers in the United States, 1975-2010. JAMA Surg 150:17-22

5. Giardiello FM, Allen JI, Axilbund JE et al (2014) Guidelines on genetic evaluation and management of Lynch syndrome: a consensus statement by the US Multi-society Task Force on colorectal cancer. Am J Gastroenterol 109:1159-1179

6. Parry S, Win AK, Parry B et al (2011) Metachronous colorectal cancer risk for mismatch repair gene mutation carriers: the advantage of more extensive colon surgery. Gut 60:950-957
7. de Vos tot Nederveen Cappel WH, Nagengast FM, Griffioen G et al (2002) Surveillance for hereditary nonpolyposis colorectal cancer: a long-term study on 114 families. Dis Colon Rectum 45:1588-1594

8. Karlitz JJ, Hsieh MC, Liu Y et al (2015) Population-based lynch syndrome screening by microsatellite instability in patients $\leq 50$ : prevalence, testing determinants, and result availability prior to colon surgery. Am J Gastroenterol 110:948-955

9. Karlitz JJ, Blanton C, Andrews P, Chen VW, Wu XC, Fontham E (2014) Colorectal cancer incidence rates in the Louisiana acadian parishes demonstrated to be among the highest in the United States. Clin Transl Gastroenterol 5:e60

10. National Comprehensive Cancer Network. Genetic/familial highrisk assessment colorectal (version 2.2015). http://www.nccn.org/ professionals/physician_gls/f_guidelines.asp. Accessed on 6 Jan 2016

11. You YN, Chua HK, Nelson H, Hassan I, Barnes SA, Harrington J (2008) Segmental vs extended colectomy: measurable differences in morbidity, function and quality of life. Dis Colon Rectum 51:1036-1043 